\title{
PRECISE COSMIC RAYS MEASUREMENTS WITH PAMELA
}

\section{A. Bruno ${ }^{a, *}$, O. Adriani ${ }^{b, c}$, G. C. Barbarino ${ }^{d, e}$, G. A. Bazileviskaya ${ }^{f}$,} R. Bellotti ${ }^{a, g}$, M. Boezio ${ }^{h}$, E. A. Bogomolov ${ }^{i}$, M. Bongi $^{c}$, V. Bonvicini ${ }^{h}$, S. Borisov ${ }^{j, k, l}$, S. Bottai ${ }^{c}$, F. Cafagna $^{a}$, D. Campana ${ }^{e}$, R. Carbone ${ }^{e, k}$, P. Carlson ${ }^{m}$, M. Casolino ${ }^{j}$, G. Castellini ${ }^{n}$,

L. Consiglio ${ }^{e}$, M. P. De Pascale ${ }^{j, k}$, C. De Santis ${ }^{j, k}$, N. De Simone ${ }^{j, k}$, V. Di Felice ${ }^{j}$, A. M. Galper ${ }^{l}$, W. Gillard ${ }^{m}$, L. Grishantseva ${ }^{l}$, G. Jerse ${ }^{h, o}$, A. V. Karelin ${ }^{l}$, M. D. Kheymits', S. V. Koldashov' ${ }^{l}$, S. Y. Krutkovi, A. N. Kvashnin ${ }^{i}$, A. Leonov', V. Malakhov, L. Marcelli ${ }^{j}$, A. G. Mayorov ${ }^{l}$, W. Menn $^{p}$, V. V. Mikhailov' ${ }^{l}$, E. Mocchiutti ${ }^{h}$, A. Monaco ${ }^{a, c}$, N. Mori ${ }^{b, c}$, N. Nikonovi, ${ }^{i, j}$, G. Osteria ${ }^{e}$,

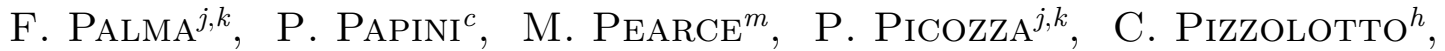
M. Ricci ${ }^{q}$, S. B. Ricciarini ${ }^{c}$, L. Rossetto ${ }^{m}$, R. SarkaR ${ }^{h}$, M. Simon $^{p}$, R. Sparvoli ${ }^{j, k}$, P. Spillantini ${ }^{b, c}$, Y. I. StozhKov ${ }^{f}$, A. VACChi ${ }^{h}$,

E. Vannuccini ${ }^{c}$, G. Vasilyev ${ }^{i}$, S. A. Voronov ${ }^{l}$, Y. T. Yurkin ${ }^{l}$, J. Wu ${ }^{m}$, G. ZAMPA ${ }^{h}$, N. ZAMPA ${ }^{h}$, V. G. ZVEREV ${ }^{l}$

${ }^{a}$ INFN, Sezione di Bari, I-70126 Bari, Italy

${ }^{b}$ University of Florence, Department of Physics, I-50019 Sesto Fiorentino, Florence, Italy

${ }^{c}$ INFN, Sezione di Florence, I-50019 Sesto Fiorentino, Florence, Italy

${ }^{d}$ University of Naples "Federico II", Department of Physics, I-80126 Naples, Italy

e INFN, Sezione di Naples, I-80126 Naples, Italy

${ }^{f}$ Lebedev Physical Institute, RU-119991, Moscow, Russia

$g$ University of Bari, Department of Physics, I-70126 Bari, Italy

${ }^{h}$ INFN, Sezione di Trieste, I-34149 Trieste, Italy

$i$ Ioffe Physical Technical Institute, RU-194021 St. Petersburg, Russia

$j$ INFN, Sezione di Rome "Tor Vergata", I-00133 Rome, Italy

"University of Rome "Tor Vergata", Department of Physics, I-00133 Rome, Italy

${ }^{l}$ NRNU MEPhI, RU-115409 Moscow, Russia

${ }^{m}$ KTH, Department of Physics, and the Oskar Klein Centre for Cosmoparticle Physics, AlbaNova University Centre, SE-10691 Stockholm, Sweden

${ }^{n}$ IFAC, I-50019 Sesto Fiorentino, Florence, Italy

${ }^{\circ}$ University of Trieste, Department of Physics, I-34147 Trieste, Italy

$p$ Universitat Siegen, Department of Physics, D-57068 Siegen, Germany

${ }^{q}$ INFN, Laboratori Nazionali di Frascati, Via Enrico Fermi 40, I-00044 Frascati, Italy.

* corresponding author: alessandro.bruno@ba.infn.it

Abstract. The PAMELA experiment was launched on board the Resurs-DK1 satellite on June 15th 2006. The apparatus was designed to conduct precision studies of charged cosmic radiation over a wide energy range, from tens of $\mathrm{MeV}$ up to several hundred $\mathrm{GeV}$, with unprecedented statistics. In five years of continuous data taking in space, PAMELA accurately measured the energy spectra of cosmic ray antiprotons and positrons, as well as protons, electrons and light nuclei, sometimes providing data in unexplored energetic regions. These important results have shed new light in several astrophysical fields like: an indirect search for Dark Matter, a search for cosmological antimatter (anti-Helium), and the validation of acceleration, transport and secondary production models of cosmic rays in the Galaxy. Some of the most important items of Solar and Magnetospheric physics were also investigated. Here we present the most recent results obtained by the PAMELA experiment.

KEYWORDS: cosmic rays, dark matter, antimatter, solar modulation, trapped radiation. 


\section{INTRODUCTION: THE PAMELA MISSION}

PAMELA - a "Payload for Antimatter Matter Exploration and Light-nuclei Astrophysics" [1] - is a satellite-borne experiment conceived to study charged particles in the cosmic radiation in a wide energy interval, ranging from several tens of $\mathrm{MeV}$ to some hundreds of $\mathrm{GeV}$, and with unprecedented precision and sensitivity. It has been in orbit since June 15th 2006 when it was launched from the Baikonur cosmodrome on board the Resurs-DK1 Russian satellite. PAMELA has been continuously taking data for more than 6 years, corresponding to $>10^{9}$ registered triggers and $>25 \mathrm{~TB}$ of down-linked data. A detailed description of the apparatus and of methodologies involved in the data analysis can be found in publications [1] 8 .

PAMELA was designed and optimized to measure the rare antimatter component in the cosmic radiation. Antiprotons and positrons, assumed to be created mostly in the interaction of cosmic rays (CRs) with the interstellar medium, are fundamental in studies of the production and propagation of CRs in the Galaxy and, together with electrons $\mathrm{e}^{-}$, provide significant details not available from the investigation of the nuclear CR component.

Above all, CR antiparticle measurements have long been considered as one of the most promising tools for indirect Dark Matter (DM) searches. Predicted $\bar{p}$ and $\mathrm{e}^{+}$fluxes from DM particles could be detectable above the background from nuclear interactions through a distortion of the measured spectra.

PAMELA also investigates the global matter/antimatter symmetry of the Universe. In case of the existence of anti-galaxies, signals of antimatter $(Z \geq 2)$ could be detected in the extragalactic radiation. PAMELA design sensitivity allows the $\overline{\mathrm{He}} / \mathrm{He}$ flux ratio to be accurately measured in a wide rigidity range.

Protons and Helium nuclei constitute the most abundant CR components, providing fundamental information to understand the origin and propagation of CR. PAMELA is able to measure their spectra with high precision in the largest explored interval, significantly constraining models.

Finally, concomitant PAMELA scientific goals include the investigation of solar modulation of CR (anti)particles during the 24th solar minimum, and the study of the geomagnetically trapped radiation.

\section{PAMELA RESULTS}

\subsection{Antiparticles}

\subsubsection{AnTIPROTONS}

PAMELA provided precise antiproton measurements in the kinetic energy range $60 \mathrm{MeV} \div 180 \mathrm{GeV}$ [2, 3], significantly improving data by previous experiments, thanks to the high statistical significance and the large explored interval. Results about the $\bar{p}$ spectrum and
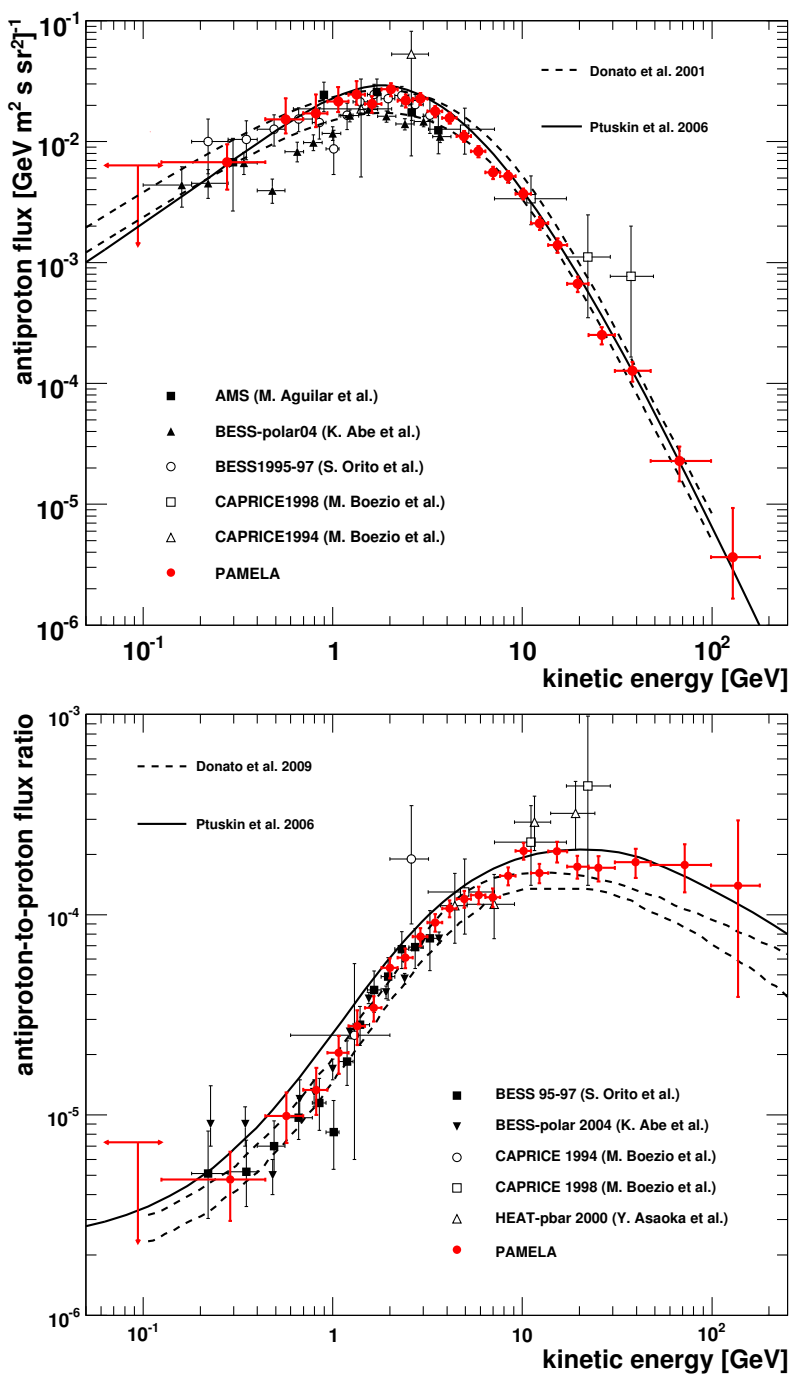

Figure 1. The $\overline{\mathrm{p}}$ spectrum (top) and the $\overline{\mathrm{p}} / \mathrm{p}$ flux ratio (bottom) measured by PAMELA, compared with data from other contemporary experiments and calculations for purely secondary production of antiprotons in the Galaxy [3, and references therein].

the $\bar{p} / p$ ratio are reported in Fig. 1, with data from contemporary experiments and some theoretical calculations for a pure secondary production of antiprotons during the propagation of CRs in the Galaxy [3, and references therein].

\subsubsection{POSITRONS}

Figures 2 and 3 report the positron fraction $\mathrm{e}^{+} /\left(\mathrm{e}^{+}+\right.$ $\left.\mathrm{e}^{-}\right)$and the positron spectrum measured by PAMELA between $1.5 \div 100 \mathrm{GeV}$. The data are compared with data from other contemporary experiments and predictions of a secondary production model [4, 5, and references therein]. PAMELA measurements cover a large energy interval, significantly reducing experimental uncertainties.

\subsubsection{Discussion}

PAMELA antiproton data reproduce the expected peak around $\sim 2 \mathrm{GeV}$ in the antiproton spectrum, 


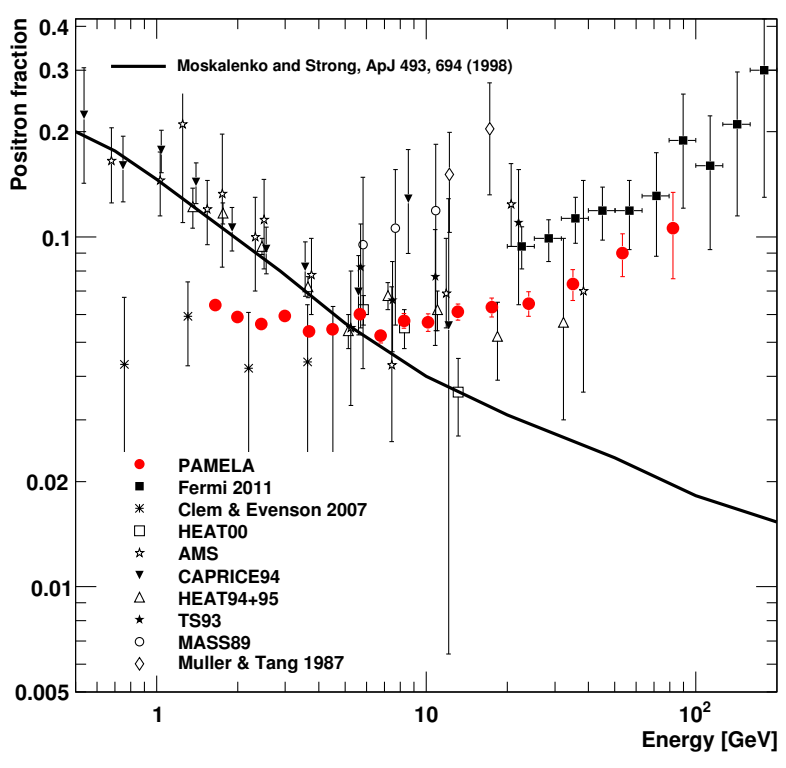

Figure 2. The PAMELA positron fraction $\mathrm{e}^{+} /\left(\mathrm{e}^{+}+\right.$ $\left.\mathrm{e}^{-}\right)$compared with other experimental data and with predictions of a secondary production model [4, 5, and references therein].

and appear to be consistent with pure secondary calculations, excluding an appreciable contribution from exotic processes in that energy range. On the other hand, PAMELA positron measurements exhibit significant features. The discrepancy with previous experimental positron data at low energy $(<5 \mathrm{GeV})$ can be interpreted as a consequence of charge dependent solar modulation effects, affecting positrons and electrons differently. Above $\sim 5 \mathrm{GeV}$ the measured positron fraction significantly deviates from predictions from secondary production models, increasing with energy. Other experimental data in this range, while affected by too large uncertainties to draw any meaningful conclusions, are consistent with the excess which is clearly shown by PAMELA.

Such unexpected rising positron fraction has triggered a considerable amount of possible interpretations based on the existence of some standard or exotic primary sources. These models are significantly constrained by antiproton data which, contrarily, appear to be in agreement with predictions of a purely secondary production. Further limits are provided by the measurement of diffuse gamma rays.

Even when the large theoretical uncertainties affecting positron fraction estimations [10] are taken into account, the presence of an excess appears manifest and consistent. As already proposed several years ago [11, a possible enhancement of the $\mathrm{e}^{ \pm}$flux could be explained by astrophysical sources like nearby pulsars (e.g, see [12 14]). No sizeable contribution from antiprotons is predicted, while counterparts in $\gamma$-rays are expected.

Alternatively, positrons can be created as secondary products of hadronic interactions inside supernova remnants (SNRs). The secondary production takes

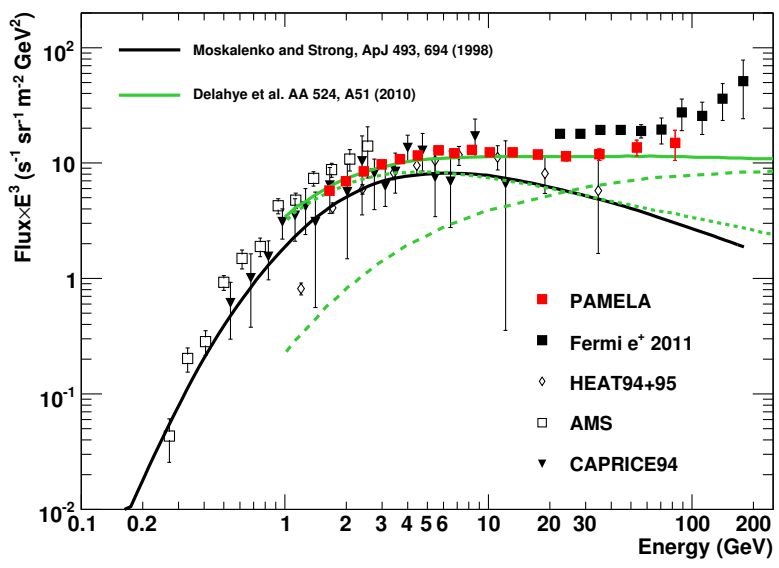

Figure 3. PAMELA preliminary results on the positron spectrum, compared with other experimental data and with predictions of a standard secondary production model [9], and with a recent calculation assuming additional primary $\mathrm{e}^{ \pm}$sources [14.

place in the same region where CRs are being accelerated. Old SNRs appear the best candidates [15]. However, according to this scenario, counterparts in the $\gamma$-rays and in the antiproton channels are expected [16], and an increase in the Boron/Carbon ratio should be observed at high energy [17].

The DM possibility, with annihilations in the halo of the Milky Way providing the anomalous antiparticle flux, is of great interest from the particle physics viewpoint. Minimal DM models can give a reasonably good fit to the PAMELA positron data, while antiprotons data put strong constraints on DM annihilations, disfavoring channels with gauge bosons, Higgs bosons or quarks. Nevertheless, the required hard spectrum would result by combining a very high DM particle mass $(\sim 1 \div 10 \mathrm{TeV})$ and a very efficient enhancement mechanism for the annihilation into charged gauge bosons [18. Further possibilities are provided by DM models assuming a dominant leptonic channel, which can fit PAMELA positron and antiproton measurements as well [12, 18. Alternatively, wino-like neutralinos [19], Kaluza-Klein particles [20], and possibly radiative corrections [21] were proposed as candidates.

\subsection{Electrons}

The electron $\left(\mathrm{e}^{-}\right)$spectrum measured by PAMELA in the kinetic energy interval $1 \div 625 \mathrm{GeV}$ is shown in Fig. 4, together with other recent measurements of the electron, and the electron plus positron $\left(\mathrm{e}^{-}+\right.$ $\mathrm{e}^{+}$) flux [6, and references therein]. PAMELA data cover the largest energy range ever achieved, with no atmospheric overburden.

\subsubsection{Discussion}

Discrepancies at low energies are partially due to solar modulation effects. Results do not show any significant spectral features and can be interpreted 


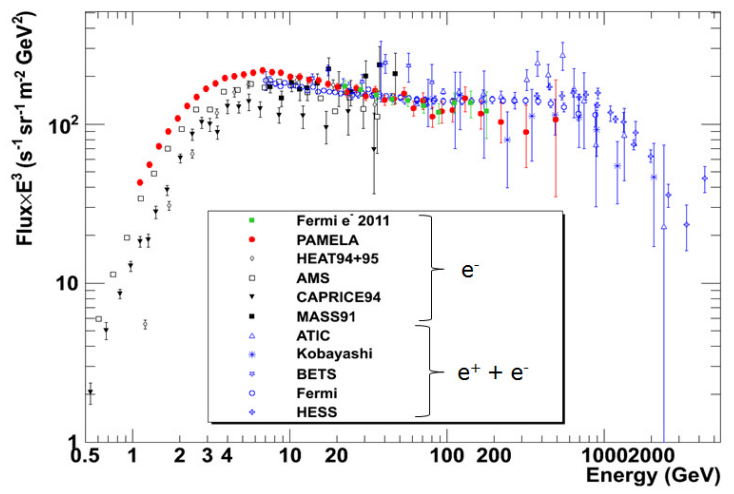

Figure 4. The PAMELA electron $\left(\mathrm{e}^{-}\right)$spectrum compared with recent electron and electron plus positron $\left(\mathrm{e}^{-}+\mathrm{e}^{+}\right)$data [6, and references therein].

in terms of conventional diffusive propagation models. Regardless of the softer spectrum, no significant disagreement results with measurements of ATIC and Fermi experiments. Existing data are also consistent with calculations including new CR sources that could explain the growing positron component [14].

\subsection{Protons and Helium nuclei}

Protons and Helium nuclei represent by far the most abundant components of the cosmic radiation. Their measurement constrains models of the CR origin and propagation in the Galaxy. The PAMELA collaboration has recently published an accurate measurement of proton and Helium spectra, in the rigidity range between $1 \mathrm{GV}$ and 1.2 TV. Results are shown in Fig. 5 together with a compilation of recent measurements. PAMELA data are consistent with those of other experiments, considering the statistical and systematic uncertainties of the various experiments [7, and references therein].

To gain a better understanding of the results, $\mathrm{H}$ and $\mathrm{He}$ data were also analyzed in terms of rigidity instead of kinetic energy per nucleon (see Fig. 6). Some important features can be drawn. Firstly, the proton and Helium spectra are characterized by significantly different spectral indices $\left(\Delta \gamma^{R}=\gamma_{\mathrm{H}}^{R}-\gamma_{\mathrm{He}}^{R}=\right.$ $.101 \pm 0.0014$ (stat) \pm 0.0001 (sys)). This aspect is also evident in Fig. 7] where the proton-to-Helium flux ratio is reported as a function of rigidity: it decreases smoothly with increasing rigidity. Moreover, PAMELA data significantly differ from a pure single power law model. The spectra gradually soften in the rigidity range $30 \div 230 \mathrm{GV}$, and at $230 \div 240 \mathrm{GV}$ they exhibit an abrupt spectral hardening (see Fig. 8).

\subsubsection{Discussion}

While differences with experiments at low energies $(<30 \mathrm{GeV})$ are explainable in terms of solar modulation effects, the hardening in the spectra observed by PAMELA around 200 GV challenges the standard CR

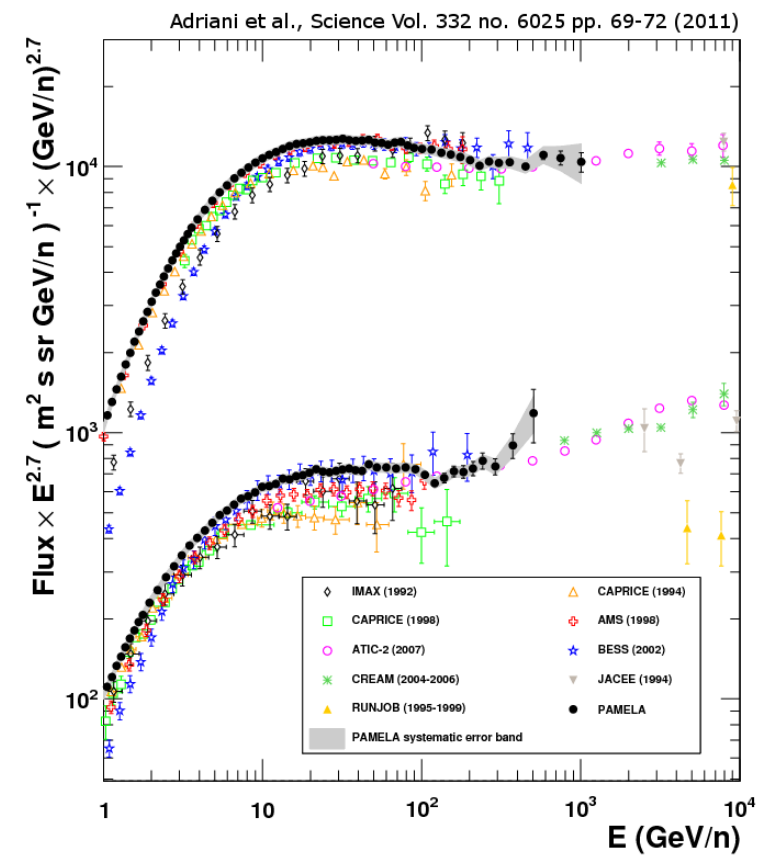

Figure 5. Proton and Helium absolute fluxes measured by PAMELA above $1 \mathrm{GeV} / \mathrm{n}$, compared with a few of the previous measurementsr [7, and references therein]. The shaded area represents the estimated systematic uncertainty.

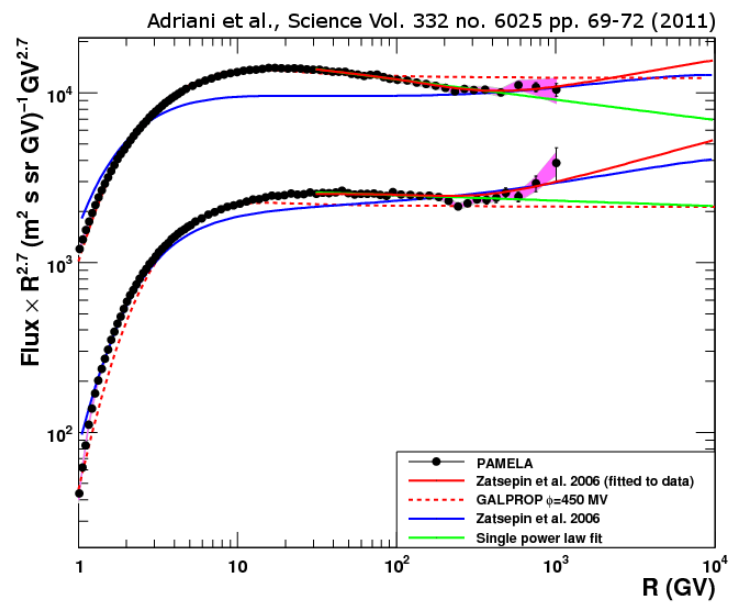

Figure 6. Proton (top points) and Helium (bottom points) data measured by PAMELA in the rigidity range $1 \mathrm{GV} \div 1.2 \mathrm{TV}$ [7, and references therein]. The lines represent the fit with a single power law and the Galprop 23 and Zatsepin\&Sokolskaya 22 models.

scenario, and could be an indication of different populations of CR sources. As an example of a multi-source model, in Figs 6 and 7PAMELA results are compared with a calculation by Zatsepin\&Sokolskaya [22, which assumes novae stars and explosions in super-bubbles as additional CR sources. Blue and red curves denote fits obtained by tuning model parameters in order to match ATIC-2 [24] and PAMELA data, respec- 


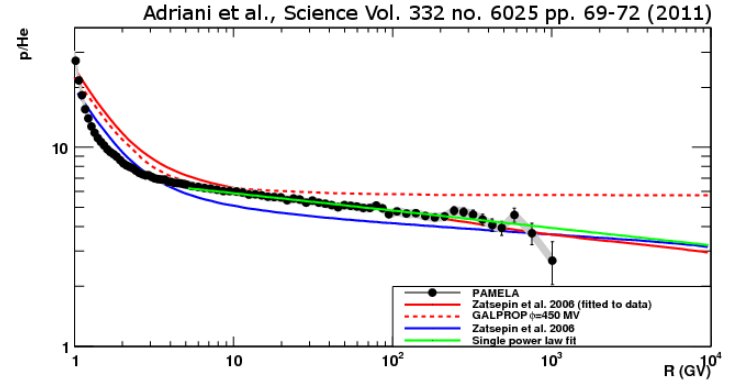

Figure 7. The proton-to-Helium flux ratio measured by PAMELA as a function of rigidity 7 . The shaded area represents the estimated systematic uncertainty. Lines show the fit using one single power law (describing the difference of the two spectral indices), the Galprop 23] and Zatsepin\&Sokolskaya models with the original values of the paper [22] and fitted to the data.
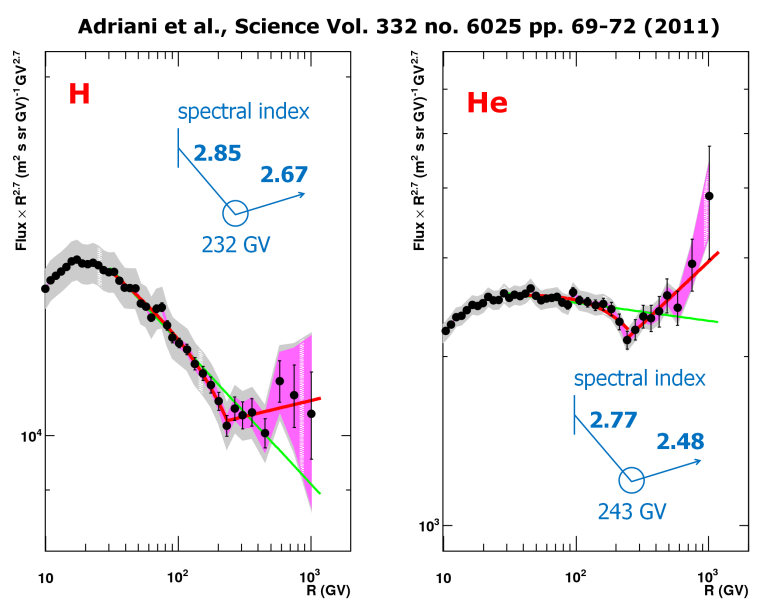

Figure 8. Proton (left panel) and Helium (right panel) spectra in the range $10 \mathrm{GV} \div 1.2 \mathrm{TV}$ [7. The shaded area represents the estimated systematic uncertainty, the pink shaded area represents the contribution due to tracker alignment. The straight (green) lines represent fits with a single power law in the rigidity range $30 \div 240 \mathrm{GV}$. The red curves represent the fit with a rigidity dependent power law $(30 \div 240 \mathrm{GV})$ and with a single power law above $240 \mathrm{GV}$.

tively. Indeed, similar results were also reported by the CREAM experiment, which observed a change of the slope for nuclei $(Z>3)$ but at a higher rigidity than the PAMELA break in the Helium spectrum [25].

\subsection{Anti-Helium}

PAMELA also places constraints on the existence of cosmologically significant amounts of antimatter, by searching for anti-Helium nuclei in the cosmic radiation. PAMELA is able to investigate the $\overline{\mathrm{He}} / \mathrm{He}$ ratio in the largest rigidity interval ever achieved, extending the measurement up to several hundreds of GV. This is particular relevant, since the predicted $\overline{\mathrm{He}}$ flux is expected to be strongly suppressed below a few GV, where most of the measurements were taken. Prelimi-
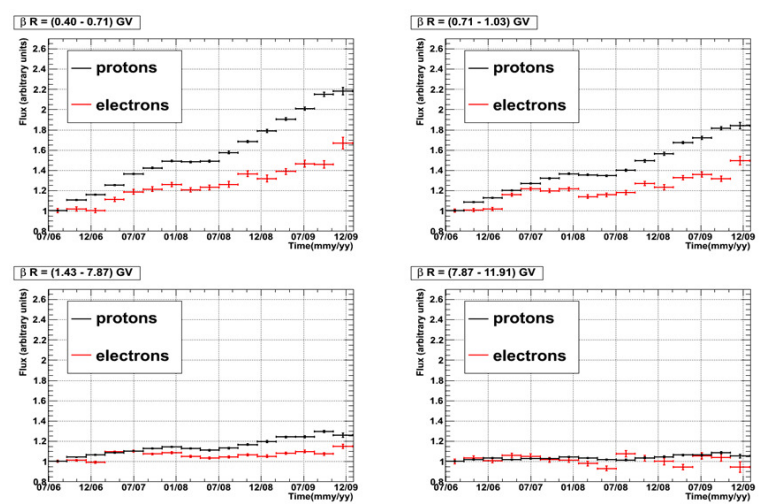

FiguRE 9. Time variations of proton (black) and electron (red) fluxes, measured by PAMELA between July 2006 and December 2009. Data (arbitrary units) are normalized to July 2006.

nary results in the rigidity range $0.6 \div 600 \mathrm{GV}$ have been provided, allowing an upper limit of $10^{-7} \div 10^{-6}$ to be put on the $\overline{\mathrm{He}} / \mathrm{He}$ ratio.

\subsection{SOLAR MODULATION}

CRs entering the heliosphere are affected by the solar wind, a continuous flow of plasma (with speed $\sim 350 \mathrm{~km} / \mathrm{s}$ ) from the sun corona carrying the solar magnetic field out into the solar system. The CR spectra variations depend on the solar activity, and this effect is called solar modulation. The solar activity has a period of about 11 years, and at each maximum the polarity of the solar magnetic field reverses. Thus, precise measurements of the energy spectra of a variety of $\mathrm{CR}$ particles in a wide rigidity range from a few hundred MV to tens of GV (where modulation effects are stronger) provide information on the interstellar spectra and the effect of the solar modulation on charge particles of both signs. PAMELA data analysis is based on data collected from July 2006 till December 2009, a period of solar minimum with negative phase $(A<0)$. As preliminary results, Fig. 9 shows the time variations of protons and electrons (arbitrary units), normalized to July 2006 data; data are provided for different intervals of $\beta R$, where $\beta$ and $R$ denote the particle velocity and its rigidity, respectively. The interpretation of PAMELA measurements needs more complex models of the propagation of CR into the heliosphere, invoking possible charge-sign dependent effects, affecting positively and negatively charged particles in different ways, depending on the solar polarity.

\subsection{Geomagnetically trapped ANTIPROTONS}

Thanks to the satellite orbit $\left(70^{\circ}\right.$ inclination and $350 \div 610 \mathrm{~km}$ altitude) PAMELA is able to measure in detail the cosmic radiation in different regions of the terrestrial magnetosphere. In particular, the spacecraft orbit passes through the South Atlantic Anomaly (SAA), allowing the observation of geomagnetically 


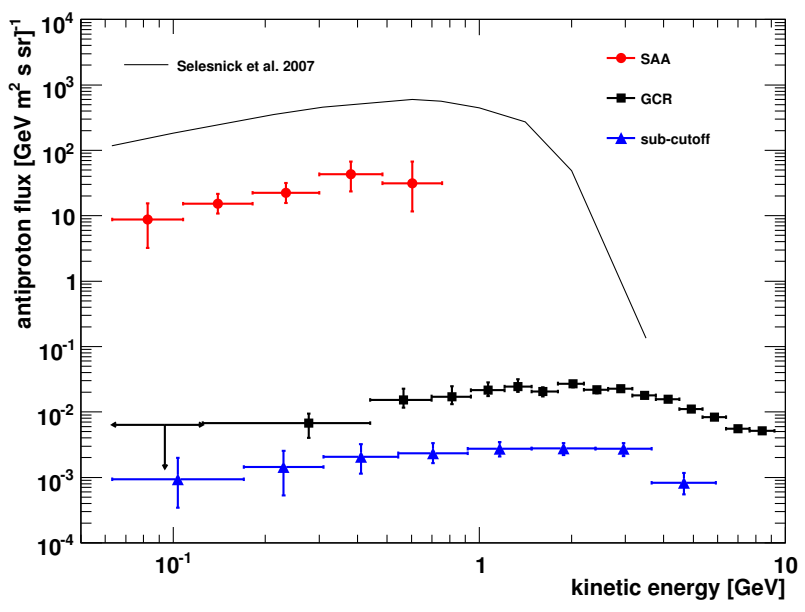

FIGURE 10 . The geomagnetically trapped $\bar{p}$ spectrum measured by PAMELA in the SAA region (red circles), compared with the mean under-cutoff antiproton spectrum outside radiation belts (blue triangles) [8, and the interplanetary $\mathrm{CR}$ antiproton spectrum (black squares) measured by PAMELA [3], together with a trapped antiproton calculation for the PAMELA satellite orbit (solid line) [26].

trapped particles from the radiation belts. PAMELA provided the first evidence of the existence of trapped antiprotons [8]. As reported in Fig. 10, at the current solar minimum the trapped $\overline{\mathrm{p}}$ flux exceeds the galactic CR $\bar{p}$ flux and the mean under-cutoff $\bar{p}$ flux outside radiation belts by 3 and 4 orders of magnitude, respectively.

\section{Conclusions}

The PAMELA experiment has been in orbit for more than six years, measuring cosmic ray particles with high precision and in a large energetic interval. The results have significant implications in the fields of astrophysics, particle physics and cosmology.

In particular, PAMELA antiparticle data has put strong constraints for theoretical models of CR production and propagation and for the existence of exotic processes. In contrast with antiproton results, the observed unambiguous positron excess appears inconsistent with predictions of the standard cosmic ray model. Proposed scenarios invoke the existence of some standard or non-standard primary sources, or non-standard secondary production mechanisms.

Additional constraints were placed by the measurement of the electron spectrum: while results agree with predictions of conventional diffusive propagation models, they do not exclude possible primary $\mathrm{e}^{ \pm}$ contributions assumed to explain the positron fraction rise.

The measurement of proton and Helium spectra by PAMELA provides fundamental information for understand the acceleration and propagation mechanisms of cosmic rays. The observed spectral features require improved models, possibly based on the existence of different source populations.
PAMELA has provided an estimation of the $\overline{\mathrm{He}} / \mathrm{He}$ ratio in a wide rigidity range, putting new limits on the existence of cosmological antimatter. Finally, PAMELA has investigated the effect of solar modulation on the cosmic radiation, and it has also achieved significant results in the study of geomagnetically trapped particles.

\section{ACKNOWLEDGEMENTS}

We acknowledge support from the Italian Space Agency (ASI), Deutsches Zentrum für Luftund Raumfahrt (DLR), the Swedish National Space Board, the Swedish Research Council, the Russian Space Agency (Roscosmos) and the Russian Foundation for Basic Research.

\section{REFERENCES}

[1] Picozza, P., et al., Astrop. Phys. 27, 296, 2007

[2] Adriani, O., et al., Phys. Rev. Lett. 102, 051101 (2009)

[3] Adriani, O., et al., Phys. Rev. Lett. 105, 121101 (2010)

[4] Adriani, O., et al., Nature, 2009, Vol 458, Pages: 607-609

[5] Adriani, O., et al., Astropart. Phys. 34 (2010) 1-11

[6] Adriani, O., et al., Phys. Rev. Lett. 106, 201101 (2011)

[7] Adriani, O., et al., Science Vol. 332 no. 6025 pp. 69-72 (2011)

[8] Adriani, O., et al., Astrophys. J. 737:L29 (2011)

[9] Moskalenko, I. V., and Strong, A. W., Astrophys. J. 493, 694-707 (1998)

[10] Delahaye, T., et al., Astron. Astrophys. 501:821-833, 2009

[11] Boulares, A., et al., Astrophys. J. 342 807-13 (1989); Atoyan, A. M., et al., Phys. Rev. D. 52 6, 3265-3275 (1995)

[12] Grasso, D., et al. Astrop. Phys. 32 (2009)

[13] Hooper, D., Blasi, P., Serpico, P. 2009, JCAP, 1, 25

[14] Delahaye, T., et al., A\&A 524 (2010) A51

[15] Blasi, P., Phys. Rev. Lett. 103 (2009) 051104

[16] Blasi, P., and Serpico, P. D., Phys. Rev. Lett. 103 (2009) 081103

[17] Mertsch, P., and Sarkar, S. , Phys. Rev. Lett. 103 (2009) 081104

[18] Cirelli, M., et al., Nucl. Phys. B 800, 204 (2008); Cirelli, M., et al., Nucl. Phys. B 813 (2009) 1

[19] Kane, G., Lu, R., and Watson, S., Phys. Lett. B 681, 151 (2009)

[20] Hooper, D., Stebbins, A., and Zurek, K. M., 2008

[21] Bergstrom, L., Bringmann, T., and Edsjo, J., Phys. Rev. D78. 103520, 2008

[22] Zatsepin, V. I., Sokolskaya, N. V., Astron. Astrophys. 458, 1 (2006)

[23] Vladimirov, A. E., et al., arXiv:1008.3642v1 (2010)

[24] Wefel, J. P., et al., International Cosmic Ray Conference (2008), vol. 2, pp. 31-34

[25] Ahn, H. S., et al., ApJL 714, L89 (2010)

[26] Selesnick, R. S., et al., 2007, Geophys. Res. Lett., 34, 20 